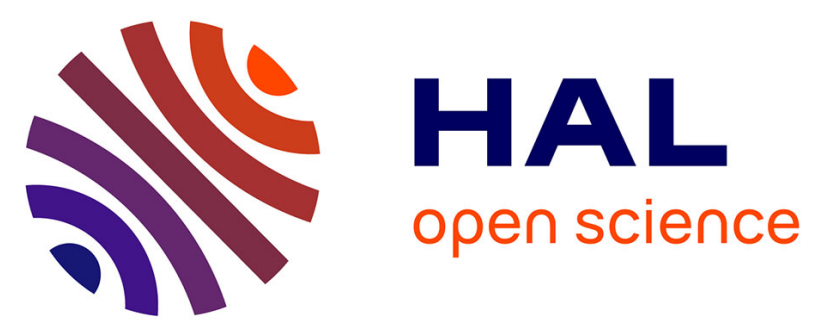

\title{
Multicolor probe-based confocal laser endomicroscopy: a new world for in vivo and real-time cellular imaging
}

Tom Vercauteren, François Doussoux, Matthieu Cazaux, Guillaume Schmid, Nicolas Linard, Marie-Amélie Durin, Hedi Gharbi, François Lacombe

\section{- To cite this version:}

Tom Vercauteren, François Doussoux, Matthieu Cazaux, Guillaume Schmid, Nicolas Linard, et al.. Multicolor probe-based confocal laser endomicroscopy: a new world for in vivo and real-time cellular imaging. SPIE BiOS - SPIE Photonics West, BiOS, Endoscopic Microscopy VIII - 2013, Feb 2013, San Francisco, United States. 10.1117/12.2002490 . hal-00800588

\section{HAL Id: hal-00800588}

\section{https://hal.inria.fr/hal-00800588}

Submitted on 14 Mar 2013

HAL is a multi-disciplinary open access archive for the deposit and dissemination of scientific research documents, whether they are published or not. The documents may come from teaching and research institutions in France or abroad, or from public or private research centers.
L'archive ouverte pluridisciplinaire HAL, est destinée au dépôt et à la diffusion de documents scientifiques de niveau recherche, publiés ou non, émanant des établissements d'enseignement et de recherche français ou étrangers, des laboratoires publics ou privés. 


\title{
Multicolor probe-based Confocal Laser Endomicroscopy: a new world for in vivo and real-time cellular imaging
}

\author{
Tom Vercauteren*, François Doussoux, Matthieu Cazaux, Guillaume Schmid, Nicolas Linard, \\ Marie-Amélie Durin, Hédi Gharbi, François Lacombe \\ Mauna Kea Technolgies, 9 rue d'Enghien, 75010 Paris, France
}

\begin{abstract}
Since its inception in the field of in vivo imaging, endomicroscopy through optical fiber bundles, or probe-based Confocal Laser Endomicroscopy (pCLE), has extensively proven the benefit of in situ and real-time examination of living tissues at the microscopic scale. By continuously increasing image quality, reducing invasiveness and improving system ergonomics, Mauna Kea Technologies has turned pCLE not only into an irreplaceable research instrument for small animal imaging, but also into an accurate clinical decision making tool with applications as diverse as gastrointestinal endoscopy, pulmonology and urology.
\end{abstract}

The current implementation of pCLE relies on a single fluorescence spectral band making different sources of in vivo information challenging to distinguish. Extending the pCLE approach to multi-color endomicroscopy therefore appears as a natural plan. Coupling simultaneous multi-laser excitation with minimally invasive, microscopic resolution, thin and flexible optics, allows the fusion of complementary and valuable biological information, thus paving the way to a combination of morphological and functional imaging.

This paper will detail the architecture of a new system, Cellvizio Dual Band, capable of video rate in vivo and in situ multi-spectral fluorescence imaging with a microscopic resolution. In its standard configuration, the system simultaneously operates at 488 and $660 \mathrm{~nm}$, where it automatically performs the necessary spectral, photometric and geometric calibrations to provide unambiguously co-registered images in real-time. The main hardware and software features, including calibration procedures and sub-micron registration algorithms, will be presented as well as a panorama of its current applications, illustrated with recent results in the field of pre-clinical imaging.

Keywords: Endomicroscopy, pCLE, Cellvizio, multi-spectral, optical fiber bundle, fluorescence, confocal, probebased Confocal Laser Endomicroscopy

\section{INTRODUCTION}

Endomicroscopy has already proven to play a key role in research and clinical imaging, thanks to its capability to provide biologists and physicians with data close to histology 12 Cellular structures can now be observed without the need for tissue sampling, thus leading to the concept of optical biopsy. Even dynamic processes, such as blood flow or neuronal activity, become observable if the involved technology can be operated in real time.

Optical Frequency-Domain Imaging (OFDI) ${ }^{3]}$ Spectrally Encoded Confocal Microscopy (SECM) ${ }^{[4}$ dual-axis microscopy, ${ }^{[5}$ and probe-based Confocal Laser Endomicroscopy ( $\mathrm{pCLE})^{2}$ are among the most widely used and described endomicroscopy technologies. All of them tackle the problem of accessing remote biological tissues with miniaturized devices, providing cellular resolution with high contrast, together with the reliability and the robustness required by medical devices standards.

The main contribution of this work is an radical evolution of probe-Based Confocal Laser Endomicroscopy (pCLE), where multiple excitations and detection channels are combined in a fiber-based microscope to provide real-time multispectral imaging, in vivo and in situ, at cellular resolution. This paper presents the versatile and modular hardware architecture and the dedicated image computing algorithms that have been developed to process raw multispectral pCLE data so as to extract only the relevant information for the end-user. The performance of our system is evaluated in vivo on several different small animal organs.

The remainder of the paper is organized as follows. A brief reminder of the principle of pCLE technology, as implemented in Cellvizio, the current flagship product of Mauna Kea Technologies, Paris, France, is given in in Section 2 together with a

*tom.vercauteren@maunakeatech.com; phone +33148241129; www.maunakeatech.com 
short review of its current applications. Section 3 emphasizes on the need for multispectral endomicroscopy. We introduce our proposed modular architecture, by concentrating on some of the main hurdles of multispectral imaging, either hardware or software in Section 4 The paper then presents in Section 5 various examples of multispectral images obtained in vivo on small animals by Cellvizio Dual Band, the first product from Mauna Kea Technologies which benefits from this new technology. Finally, Section 6 concludes the paper by providing insights on the future of this technology.

\section{PROBE-BASED CONFOCAL LASER ENDOMICROSCOPY}

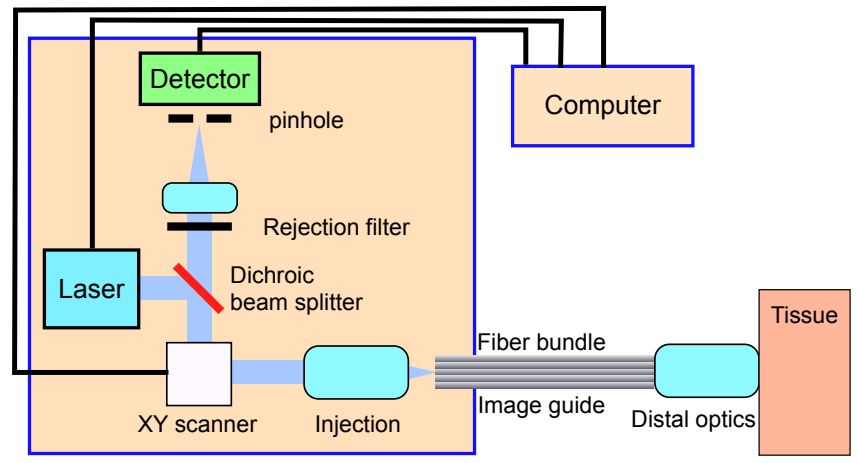

Figure 1: Current single spectral band Cellvizio architecture

Fiber-based confocal laser endomicroscopy was introduced several years ago in the field of small animal imaging $6+8$ and more recently in the clinical arena by various academic 910 and industrial $1 / 112$ actors. Though several technical approaches have been proposed, $\frac{13}{13}$ only a few of them have been developped and are now commercially available as routine instruments. Among the commercially available options, Cellvizio is a fluorescence confocal microscope which makes use of fiber bundles to access the tissue to be imaged in the living organism.

In brief, the system is a confocal microscope which makes use of a $488 \mathrm{~nm}$, or $660 \mathrm{~nm}$, excitation which is sequentially injected in tens of thousands of tiny fibers optics grouped in a fiber bundle. Excitation is conducted by the fibers down to the tissue to be examined where it is focused by some distal optics which defines the field of view (FOV), the lateral and the axial resolution of the system. Endogenous or exogenous fluorescence is then produced, which is collected by the very same individual fiber and redirected towards a single detector, most of the time an avalanche photodiode (APD). Scanning the laser onto the proximal side of the fiber bundle is performed by a combination of a resonant and a galvanometric mirror, providing an overall frame of 9 to 18 frames per second (fps) and even $200 \mathrm{fps}$ by reducing the field of view. Image processing is needed, first to compensate for fiber-to-fiber differences in transmission and background, but also to remove the well-known fiber honeycomb pattern and reconstruct a smooth and readable image. Figure 2 illustrates the impact of

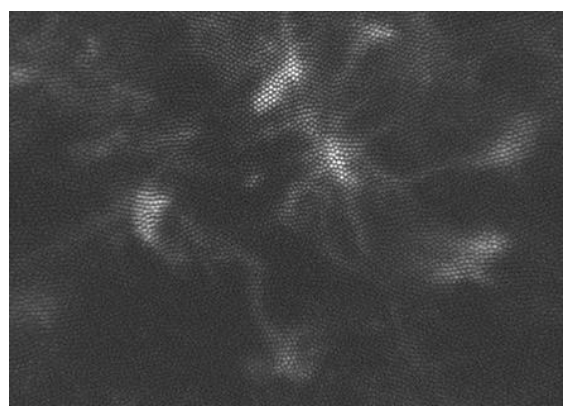

(a) Raw image

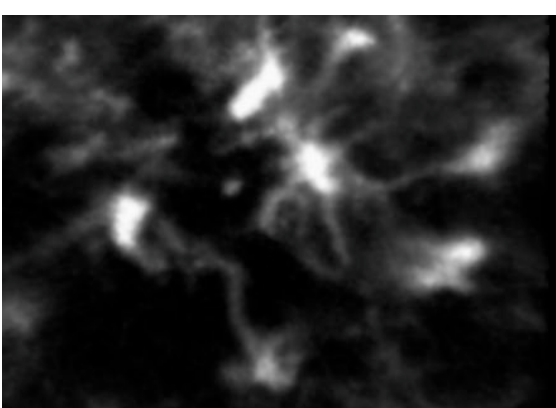

(b) Reconstructed image

Figure 2: A typical example of the image reconstruction applied to a single spectral band Cellvizio raw image. The mouse neurons are clearly visible on the reconstructed image but not on the raw one. The field of view is approximatively $200 \mu \mathrm{m}$ 
the image computing algorithms on raw pCLE data. Both hardware and software aspects have extensively been discribed in the literature. 14

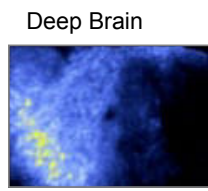

Heart

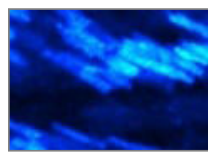

Cornea

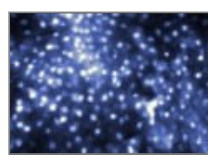

Mesentery

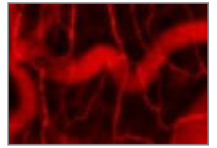

Ear

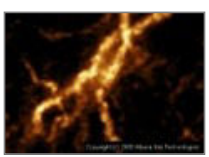

Lymph Node

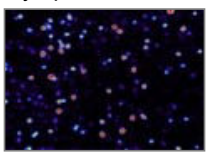

Liver

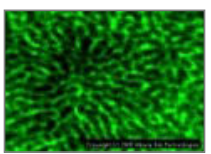

Muscle

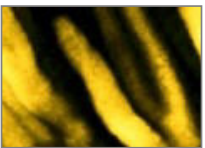

Kidney

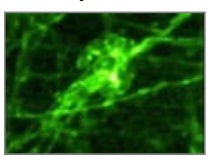

NMJ

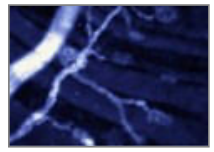

Colon

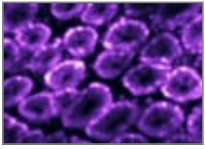

Bladder

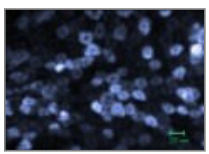

Figure 3: Cellvizio can be used for a wealth of different applications in small animal and pre-clinical imaging. We can see here images taken in many different mouse organs using fake colors

Cellvizio uses a proprietary hardware and software which provide researchers and clinicians with real-time (up to 200 fps), high-resolution (down to $1.2 \mu \mathrm{m}$ ) images with a FOV ranging from 240 to $600 \mu \mathrm{m}$ in diameter. A number of software features $\frac{18}{18}$ also permit, for research applications, to analyse data in real-time (histogram, temporal statistics in multiple regions of interest), or process them off-line: vessel segmentation, functional capillary density (FCD) analysis, mosaicing, etc. As far as clinical applications are concerned, video mosaicing, reviewing recent images, selecting sub-sequences to be archived, and searching patient data ${ }^{19}$ is also possible.

As shown in Fig. 3, research applications have covered the fields of neuroscience (deep brain imaging on awake animals), cancer research, reproduction and stems cells, among others. In the clinic, Fig. 4 illustrates that Cellvizo is routinely used in gastrointestinal (GI) endoscopy (esophagus, colon, biliary and pancreatic ducts) and pulmonology (proximal and distal airways, alveoli).
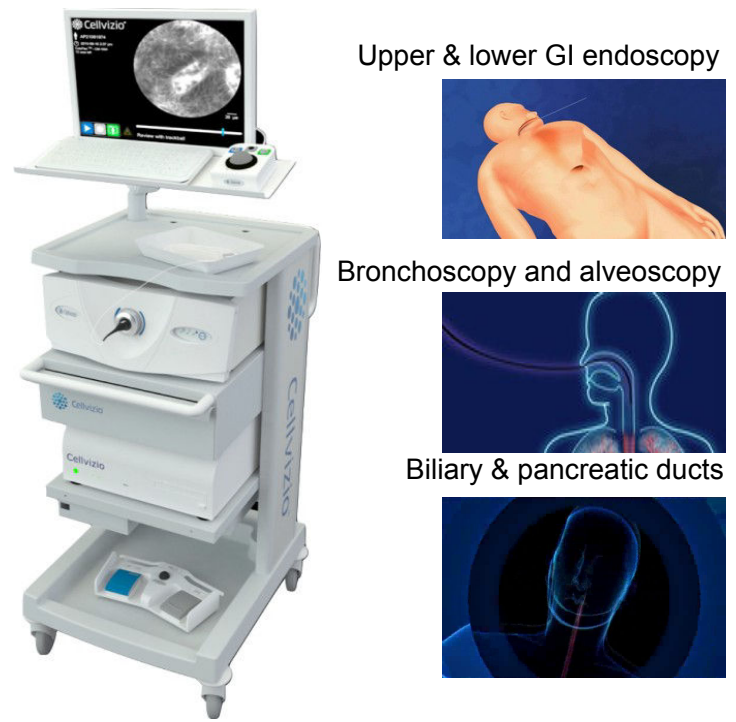

Bronchoscopy and alveoscopy

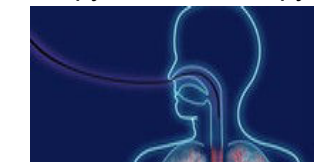

Biliary \& pancreatic ducts
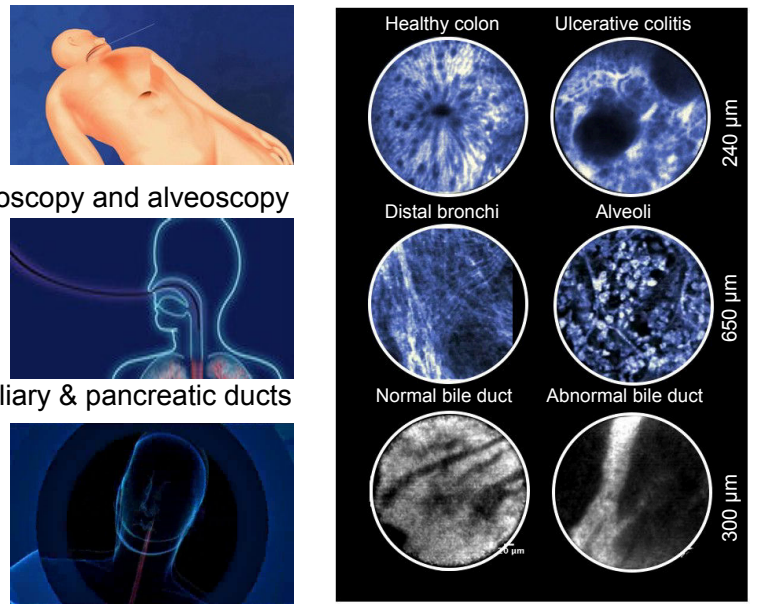

Figure 4: Overview of the current clinical applications of Cellvizio. As illustrated on the left, the user interface of the clinical system has been designed to be clutter-free and intuitive for the physicians

Based on the same Cellvizio core technology, other imaging modalities can be derived: reflectance microscopy, $\frac{16 \mid 20}{140-}$ rescence lifetime imaging microscopy (FLIM) ${ }^{21}$ when coupled with convenient detection hardware and software, or even spectroscopy $\sqrt{12}$ and two-photon imaging. ${ }^{22}$ 


\section{THE NEED FOR MULTISPECTRAL IMAGING}

Among the benefits provided by real-time in situ microscopy, of particular interest can be: following molecular interactions, studying biodistribution and coupling morphological with functional imaging. To achieve these goals, solutions similar to what is widely used on benchtop microscopes can be implemented: multiple excitations in combination with multiple fluorescent markers, targeting multiple biological entities, possibly in interaction. Again, different approaches have been proposed, providing multispectral imaging capabilities, with ${ }^{23.24}$ or without ${ }^{25}$ microscopic performances.

A common technical specification is often the simultaneous illumination and detection in multiple channels, led by a critical need for unambiguous image co-registration at different wavelengths, even at microscopic scale, and despite relatively rapid tissue motion. Such simultaneity is of course strongly demanded, for instance, when observations involve processes which are expected to last a very short time, like Förster Resonant Energy Transfer (FRET) ${ }^{26}$

In the following sections, we build upon earlier preliminary work ${ }^{27}$ and describe how Cellvizio architecture has been modified to accomodate with such new requirements, as well as the particular constraints of in vivo microscopy, such as frame rate and motion artifacts, while keeping in mind industrial standards of reliability, maintainability, and product evolution capability.

\section{A VERSATILE MULTISPECTRAL ENDOMICROSCOPE DESIGN}

\subsection{Hardware design}

\subsubsection{Optical design}

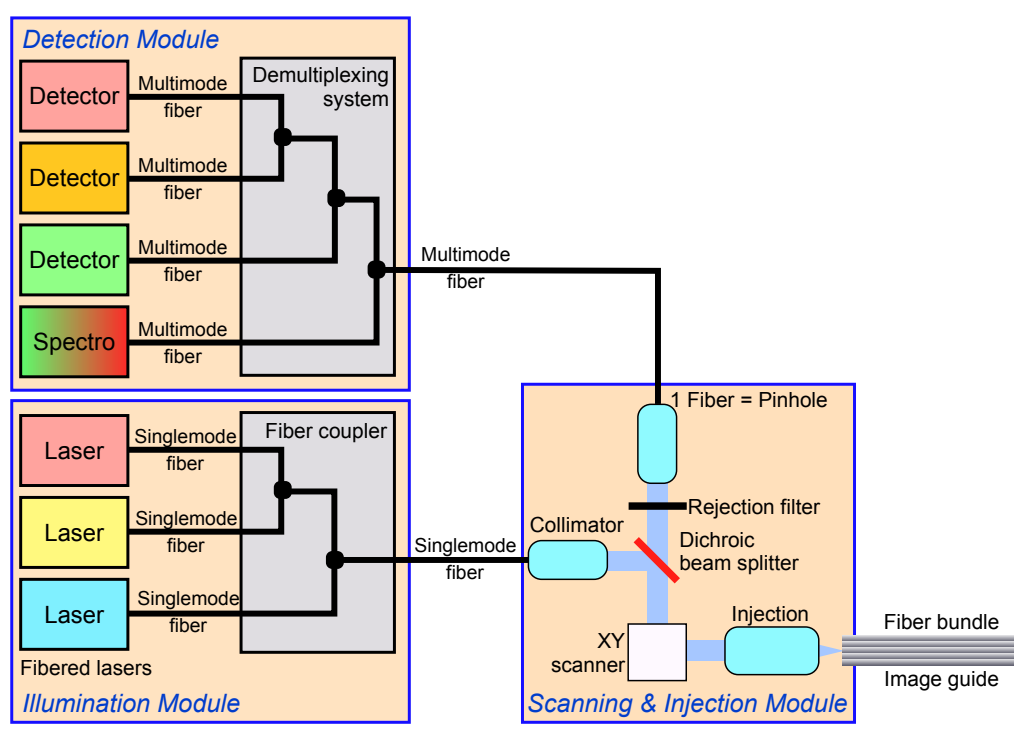

(a) Schematic representation of the proposed architecture

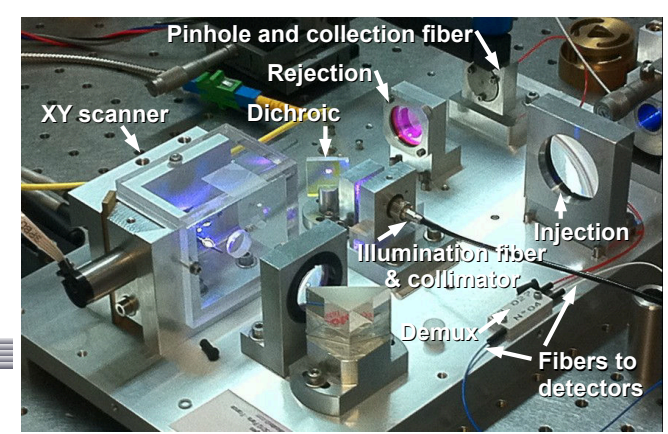

(b) Picture of the Scanning and Injection Module

Figure 5: Overview of the proposed modular multispectral endomicroscopy architecture

The most straightforward way to move towards multicolor imaging would have been to implement a second laser unit, and a second detector, within our current optical design inFig. 1. Such a solution hardly permits moving towards more than two colors. Nor does it allow developing a versatile solution where excitation wavelengths can be exchanged on demand. Furthermore, it prevents from implementing other modalities such as reflectance microscopy and spectroscopy. For this reasons, we decided to develop a modular design, illustrated in Fig. 5. where the three main functions: a) illumination, b) scanning and injection into the image guide and c) detection, are physically separated and located within different modules, all of them coupled together through optical fibers. This not only offers more flexibility for product evolution, but also permits an easier maintenance. 
The Illumination Module, first, harbors up to three pigtailed laser units, optically coupled together through a multiplexer into a singlemode fiber, which is further used as a single point illumination source in the Scanning and Injection Module. This configuration avoids any misalignement between excitation lines, which is much preferable, from an industrial standpoint. In the Cellvizio Dual Band product, two laser sources are present : 488 and $660 \mathrm{~nm}$, delivering up to $30 \mathrm{~mW}$ each.

The Scanning and Injection Module, second, includes a traditional XY scanner (resonant at $4 \mathrm{kHz}$ coupled with galvanometric at 9-18 Hz), an injection objective (numerical aperture $>0.4$, field of view $=1.2 \mathrm{~mm}$ ), the connector to the image guide, a dichroic beamsplitter (reflecting the multiple excitation lines), plus the necessary optics to collimate the illumination beam and focus the collected fluorescence onto the output multimode fiber. This fiber also acts here as a filtering pinhole with a $50 \mu \mathrm{m}$ diameter. When moving towards multiple spectral domains, the optical design must of course comply with more severe achromatism constraints than in the single spectral band case. The singlemode input fiber and the multimode output fiber must be imaged onto the same individual fiber of the bundle, at any time (i.e. anywhere in the scanned FOV), and at all wavelengths. The chromatic performance of our system is evaluated in Section 4.2.1 and is typically below $0.5 \mu \mathrm{m}$.

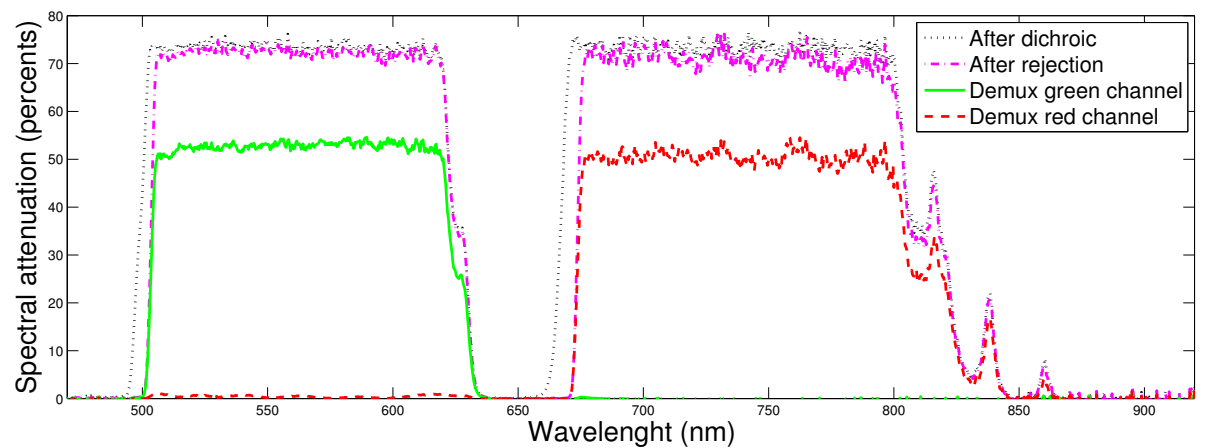

Figure 6: Evaluation of the photometric performance of our system. For a pure white light source, having a spectral density of $100 \%$, located at the focal plane of the Scanning and Injection Module, cf. Fig. 5b, our system transmits approximatively $75 \%$ of the initial spectral density after both the dichroic beam splitter and the rejection filter in the subbands of interest. For both the green and the red channel, after the demultiplexing system, we collect approximatively $55 \%$ of the initial spectral density in the subband of interest. We can also see that the wavelengths corresponding to the lasers are perfectly attenuated.

The Detection Module, finally, consists in a fiber-coupled demultiplexer separating the respective spectral bands and sending, though multimode fibers, the content of each of them to individual pigtailed APDs. In this new configuration, APDs are directly piggy-backed on the readout electronics board, thus reducing to a minimum the length of analog signal transmission and also improving the sensitivity to external noise sources. In another possible configuration, one output of the multiplexer may be sent towards a miniaturized spectrograph, many of them existing commercially. This spectrograph may then be coupled to the acquisition hardware for example via a standard USB interface.

The photometric performance of our Scanning and Injection Module and of our Detection Module is evaluated in Fig. 6 by measuring, at different locations within our optical setup, the spectral attenuation of a white light source placed at the focal plane of the Scanning and Injection Module. We observe a nice flat attenuation of $45 \%$ of the signal on the spectral bands of interest. We can also see a total attenuation at the laser wavelengths.

Laser powers can of course be adjusted independently by software control, depending on the user needs. The laser beams are always sent towards the system (and so, towards the tissue) simultaneously, not sequentially. Such a simultaneous configuration is unfortunately less efficient, in terms of transmission budget, than sequential illumination but is required to properly deal with tissue motion. Coupling the lasers and demultiplexing the fluorescence responses are not lossless solutions. The demultiplexer loss seen in Fig. 6, in particular, could easily be avoided in the case of sequential illumination (on a frame per frame basis, for instance), with a unique detector collecting all the available fluorescence, at any time, so for any excitation, and over the whole available spectral range. But the simultaneous illumination configuration is nevertheless much more convenient if not mandatory, whenever in vivo imaging is at stake. Indeed, any delay between imaging in 
different colors cannot be accepted, due to frequent and rapid relative tissue movements (at least at microscopic scale), or due to the need for observing dynamic processes.

Of course, the confocal miniprobes, displayed in Fig.7b, must also comply with the same very severe achromatism constraints, a performance already achieved by our distal optics as presented in earlier work. 28

Similar to the single spectral band case, one of the main mechanical challenges is to obtain a high coupling efficiency in a micron-sized fiber optic. For that purpose, we reuse the specific opto-mechanical connector designed to connect the confocal miniprobes to the Scanning and Injection Module of our single spectral band system. This connector gives a high repeatability in the focus position, as well as an optimal injection along the whole image field.

\subsubsection{Electronics design}

The optical design presented in the previous section relies on a 2D scanner, several lasers and several detection bands. Our system being designed for in vivo microscopy, it requires a high frame rate to acquire images that do not suffer from too large motion artifacts. Working at a frame rate between 9 and $18 \mathrm{~Hz}$ proved to be efficient for the single spectral band case. We therefore chose not to go below this range. Because of the number of elements that need to be controlled and because of the large amount of data produced by our multispectral system, new electronic boards were designed.

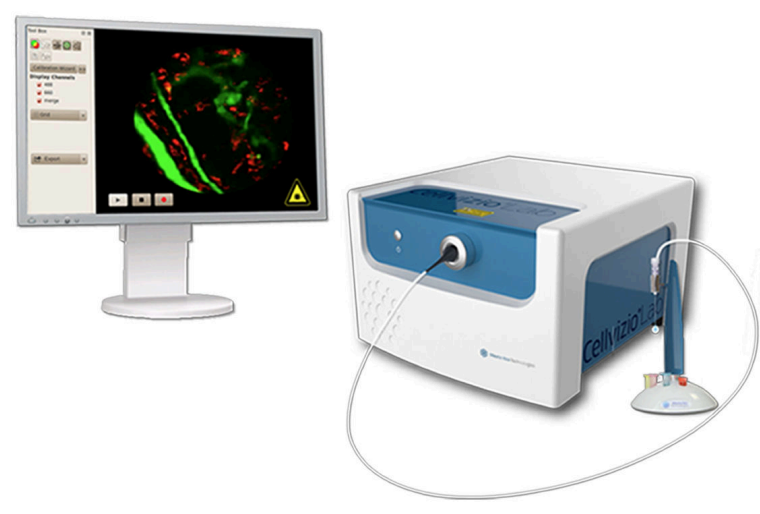

(a) Cellvizio Dual Band system

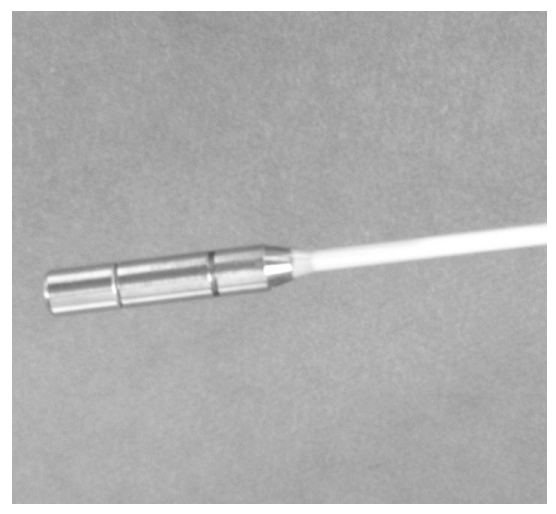

(b) Tip of the UltraM flexible optical miniprobe. It offers a lateral resolution of $1.4 \mu \mathrm{m}$, a field of view of $240 \mu \mathrm{m}$ with an outer diameter of $2.6 \mathrm{~mm}$

Figure 7: Cellvizio Dual Band is the first product dedicated to small animal and preclinical imaging based on our modular multispectral architecture presented in Fig. 5

The scan surface is adjustable, with a typical size of $600 \times 600 \mu \mathrm{m}^{2}$ corresponding to the surface of the fiber bundle we use. In order to benefit from the full possible resolution allowed by the tens of thousands fiber optics that compose our fiber bundle, we need to sample the bundle proximal side in such a way that all fibers can be isolated from each others. This led us to rely on a $80 \mathrm{~ns}$ pixel period. With this sampling rate, each fibers is sampled by approximatively 25 to 35 raw pixels hence nicely meeting the Nyquist criterion by oversampling the fiber bundle.

A customized synchronization hardware controls the mirrors and digitizes, synchronously with the scanning, the level of each pixel. The fluorescent optical signal is detected by one photodetector per spectral range. At the end of this phase, the detection board filters the signal, transmits it to low-noise amplification stages prior to performing an analog-to-digital conversion (ADC).

To ensure a proper alignement of the sampling with the scanning, the motherboard is synchronized by a synchronization signal coming from the resonant mirror. The synchronized reception of the signal is also used for the image transmission to the system computer using the isochronous packet mode of the IEEE1394 FireWire protocol. Raw data images are then available on the system computer for processing, storage and display. 


\subsection{Key software components and calibration procedure}

As mentioned in the previous section, our multispectral endomicroscope design relies on scanning, with a combination of rapid sine-shaped horizontal scanning and saw-tooth-shaped vertical scanning, the proximal side of a fiber bundle that serves as an image guide to deliver and collect ligt. Because of the fiber bundle, the system produces raw images with a strongly visible honeycomb pattern. Each fiber within the fiber bundle has unique physical properties. In addition to transmitting light, the fibers also produce autofluorescence and Raman signal that may shadow the useful signal coming from the tissue. Furthermore, the specific scanning pattern on which we rely introduce geometric distortions on the raw images and, despite the good performance of the optics, small residual chromatic aberrations may exist among the different spectral bands.

As illustrated on Fig. 11, the non-uniform honeycomb pattern and geometric distortions make the raw, unprocessed, data impractical for user interpretation or for automated analysis if left untreated. As similar problems arise in the single spectral band case, algorithms that take on the image reconstruction task in real time have already been developed at Mauna Kea Technologies to provide users with high-quality, smooth-motion video sequences in the single band case. ${ }^{14 \mid 29}$ Most of the available methods from other groups 30 - 32 are only focused on the removal of the honeycomb pattern and often only imply performing a simple low-pass filtering. The approach of Mauna Kea Technologies for the single spectral band case not only removes the honeycomb pattern but also recovers the true signal that comes back from the tissue and compensates for the geometric distortions.

In this paper, we propose a dedicated algorithm to compensate for the residual lateral chromatic aberrations across the spectral bands and adapt the single spectral band algorithm 14 to recover the tissue signal from our raw multispectral data. In addition to making the images more readable, these algorithms will allow us to apply more advanced image computing algorithms $\frac{18}{18}$ that would otherwise fail due to the artefacts present in the raw data.

\subsubsection{Geometric distortions and residual lateral chromatism calibration}

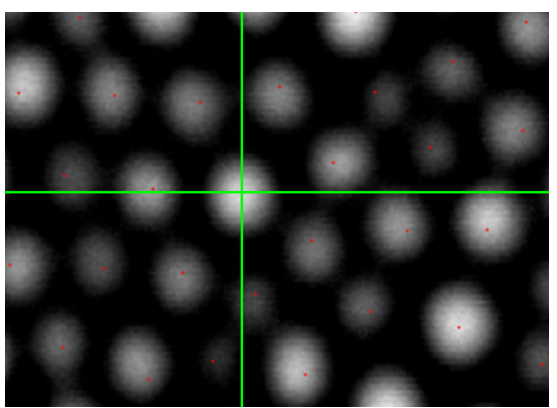

(a) $488 \mathrm{~nm}$ excitation and [500-630]nm detection range

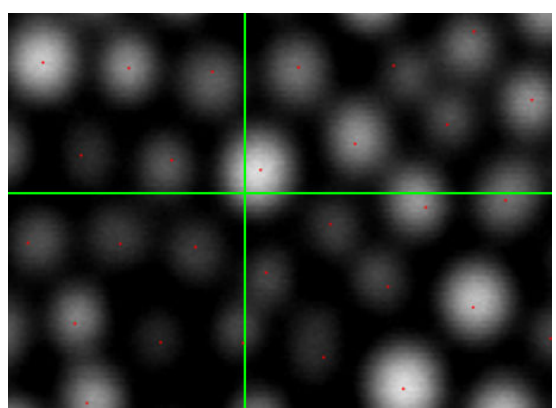

(b) $660 \mathrm{~nm}$ excitation and [680-800] $\mathrm{nm}$ detection range

Figure 8: Visualization of the residual cross-spectral band lateral chromatism. The cross displays the same image location on each spectral subband image. It is aligned with a fiber center on the green channel but is offset in the red channel

The scanning reproducibility of our system is better than one half of a fiber diameter. This performance enables the calibration of the continuous motion of the illuminating spot. The sine-wave shape of the geometric distortion due to the resonant mirror can thus be compensated. This correction should permit a comprehensive rectangular mapping of the field of view. This is mandatory for any metric interpretation using the images, or any complex combination of individual frames. In this work, we propose to adapt the distortion compensation framework that has previously been proposed for the single spectral band case ${ }^{14}$ to our multispectral case.

Although the geometric distortions are mostly created by the varying velocity of the horizontal scanning mirror, lateral chromatic aberrations also play a role. Even if their effect is limited by our optical design, Fig. 8 illustrates that residual sub-micron difference in the geometric distortions may exist across the spectral bands.

Since the total geometric distortions are spectral-band dependent, the most straightforward way of compensating them in the multispectral case would be to calibrate them independently on a per spectral band basis. Although this works to compensate gross distortions, it fails to align the different spectral bands with a sub-micron performance. 


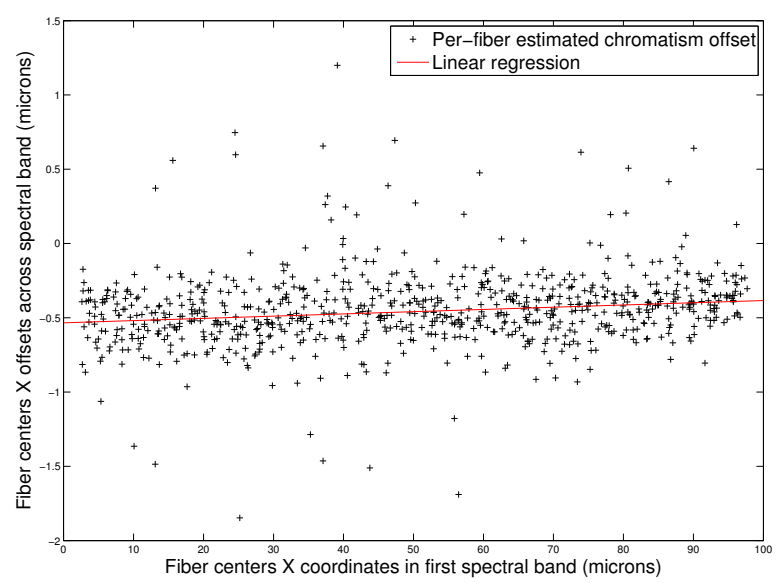

(a) Residual chromatic distortions on the horizontal $(x)$ coordinate and linear regression fit

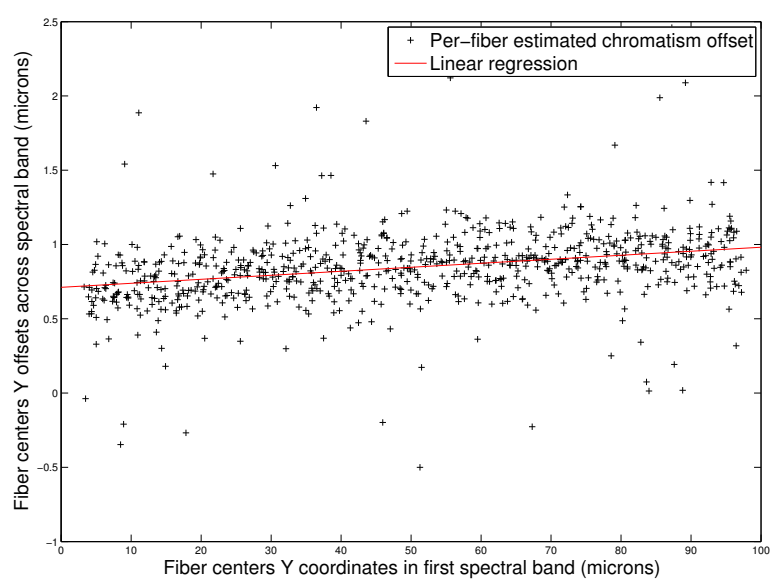

(b) Residual chromatic distortions on the vertical $(y)$ coordinate and linear regression fit

Figure 9: Evaluation of the residual chromatic distortions between the [500-630]nm detection band and the [680-800]nm detection band used as reference. A linear model provides a very good approximation of the observed residual distortion. We also see that the residual lateral chromatic distortions are within $1 \mu \mathrm{m}$

In this paper, we propose to rely on a reference spectral band that may be arbitrarily chosen and to calibrate only this reference band using the previous algorithm available for the single spectral band case. We then pre-compensate all spectral bands with the geometric distortion function estimated from the reference band. This is sufficient to compensate gross distortions and we then focus on estimating and compensating only the residual inter-band chromatism.

As a first-order approximation, the residual lateral chromatism between a spectral band $k$ and the reference band, ref, can be modeled as a linear system:

$$
\left\{\begin{array}{l}
x_{k}=a_{k}^{x} \cdot x_{\mathrm{ref}}+b_{k}^{x} \\
y_{k}=a_{k}^{y} \cdot y_{\mathrm{ref}}+b_{k}^{y}
\end{array}\right.
$$

where $x$ and $y$ are coordinates in the pre-compensated space; and $a$ and $b$ are the parameters of the residual lateral distortion.

Looking at the raw images in Fig. 11d, and Fig. 11e, it should appear clearly that even when no tissue signal is observed, the fiber pattern can appear quite differently among the different spectral bands. This is explained by the specific physical properties of each fibers. Some fiber may for example produce more autofluorescence but less Raman signal than their neighbors. This disparity leads to the fact that intensity-based image registration algorithms may have some difficulties in registering robustly and with a very high accuracy such images even when relying on multimodal similarity metrics such as the mutual information.

We therefore chose to rely on the fiber center detection algorithm developed at Mauna Kea Technologies for the single spectral band case. Our approach can basically be divided into:

1. calibration of the geometric distortion on the reference band

2. pre-compensation of the distortions on each band using the reference band distortion parameters

3. independent fiber detection on each pre-compensated spectral band

4. estimation of the residual lateral chromatic distortion by matching the detected fiber centers

5. compensating each band with the composition of the reference band geometric distortions and the residual chromatic distortion to the reference band

After these steps, the distortion-compensated raw images are co-registered and we use the fiber centers detected on the reference channel for each distortion-compensated spectral band.

Using the detected fiber centers instead of the raw images pixel intensities allow us to be quite insensitive to imaging noise and to the differences in fiber contrast across the spectral bands. To estimate the residual lateral distortion from the fiber 
centers, we designed a symmetric and robust version of the Iterative Closest Point (ICP) $\frac{\sqrt{33}}{3}$ algorithm. The symmetry and robustness are achieved by relying on an orthogonal linear regression as explained in previous work ${ }^{29}$ Although ICP is known to strongly suffer from local minima, in our case, it is not such an issue because the residual chromatic aberration are small and only lead to displacements below half the inter-core distance.

As we expect the distortion parameter $a$ of to be close to one, we typically rewrite (1) as:

$$
\left\{\begin{array}{l}
x_{k}-x_{\mathrm{ref}}=\left(a_{k}^{x}-1\right) \cdot x_{\mathrm{ref}}+b_{k}^{x} \\
y_{k}-y_{\mathrm{ref}}=\left(a_{k}^{y}-1\right) \cdot y_{\mathrm{ref}}+b_{k}^{y}
\end{array}\right.
$$

before performing the linear regression.

Figure 9 illustrates the performance of our approach. We can see that the residual lateral chromatic distortion are below $1 \mu \mathrm{m}$ and that a linear model matches the data quite well. Our robust regression procedure also properly deals with outliers arising from the fact that some fibers may be seen in one spectral band but may be hard to distinguish in the other subbands. Thanks to the symmetry of our regression procedure, our results are actually independent of the channel used as reference.

\subsubsection{Photometric calibration and image reconstruction}

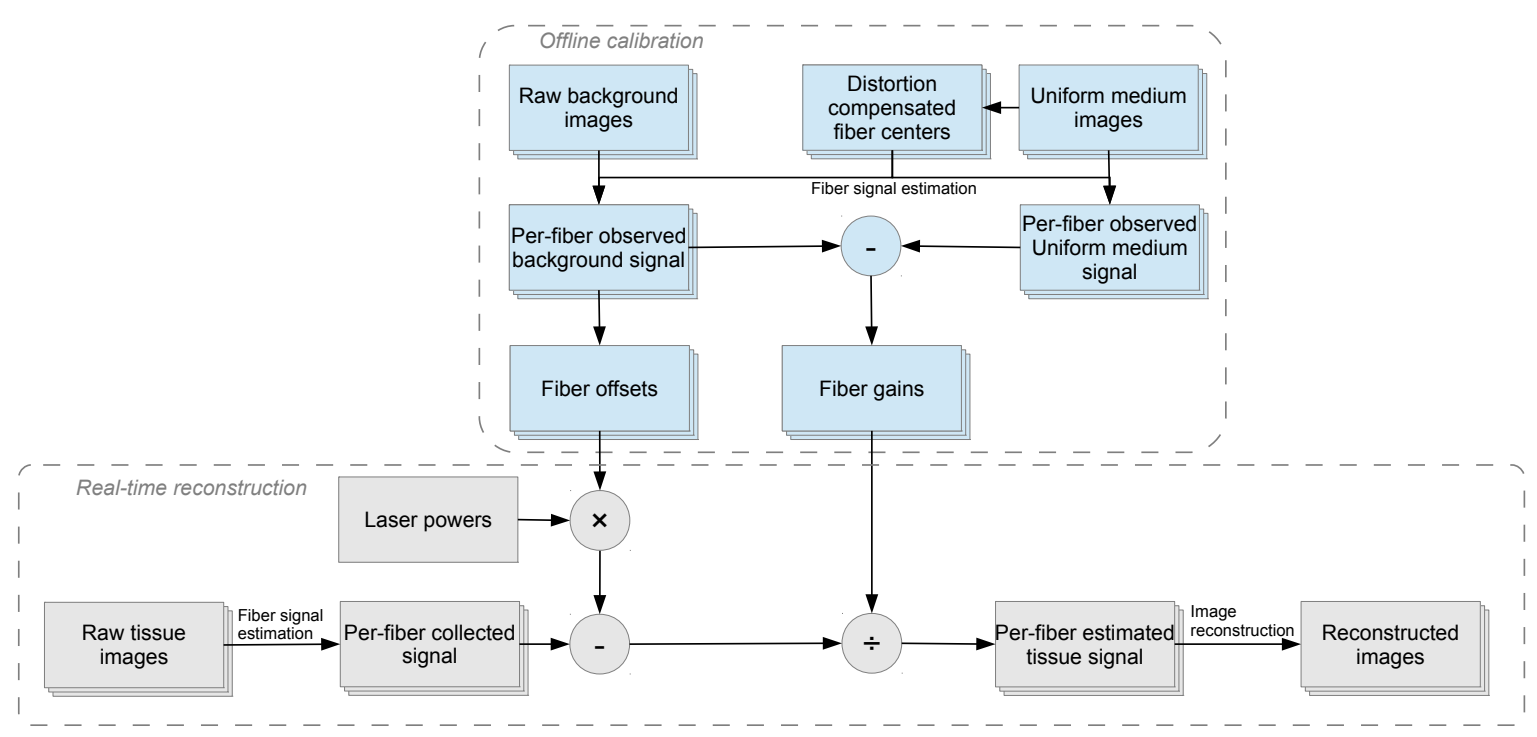

Figure 10: Schematic overview of the multispectral image computing pipeline used for image reconstruction

Thanks to the geometric distortion compensation algorithm presented in the previous section, we now have access to the distortion-compensated position of each fiber composing the fiber bundle and our estimation is consistent across spectral subbands. In this section we show how to use this information to reconstruct images displaying only the relevant information to the user, that is, a reconstructed signal proportional to the tissue signal only and exempt of any honeycomb pattern.

As in Section 4.2.1, the most straightforward approach would be to apply the algorithm developed for the single spectral band case ${ }^{14 \mid 29}$ on each spectral band independently. This would have several drawbacks: we would not account for the residual chromatic aberrations, therefore leading to reconstructed images suffering from chromatic aberrations, we would potentially detect a different number of fibers per spectral band, and it would not account for the fact than one laser may influence several spectral bands. This second argument is actually a real practical issue since calibration images will typically be acquired at full laser power while the user may choose to reduce any of the laser power anytime during use. If not taken into account, this will lead to a wrong estimation of the tissue signal.

Figure 10 illustrates the adaptation of the classical pCLE image computing pipeline to the case of multispectral data acquisition. As described in the case of a single spectral band, $[1429$ each fiber $i$ of the endomicroscopy miniprobe acts as a 
mono-pixel photodetector with an affine photometric transfer function. We therefore essentially have one sampling point per fiber. The same observation holds in the multispectral case for each spectral subband $k$ :

$$
v_{i}^{k}(t)=\alpha_{i}^{k} u_{i}^{k}(t)+\beta_{i}^{k}
$$

where

- $\alpha_{i}^{k}$ and $\beta_{i}^{k}$ are respectively the gain and offset of fiber $i$ within the spectral band $k$

- $u_{i}^{k}(t)$ is the fluorescence power of the observed medium seen at time $t$ by fiber $i$ within the spectral band $k$. In the case of a single fluorophore, $u_{i}^{k}(t)$ is simply proportional to the concentration of the fluorophore

- $v_{i}^{k}(t)$ is the signal collected at time $t$ by the fiber detector $i$ within the spectral band $k$

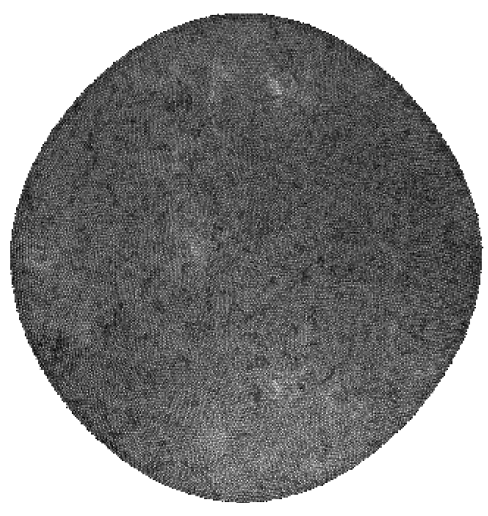

(a) [500-630]nm detection range mostly influenced by $488 \mathrm{~nm}$ excitation

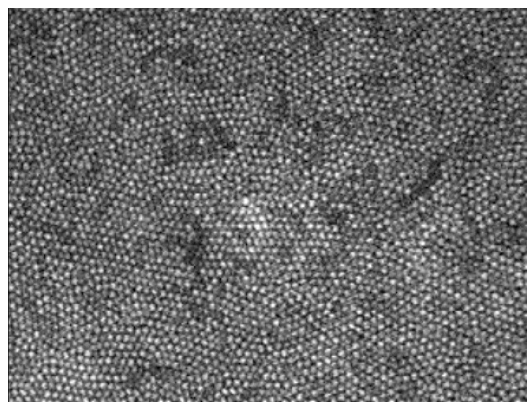

(d) Zoom on a portion of Fig. 11a

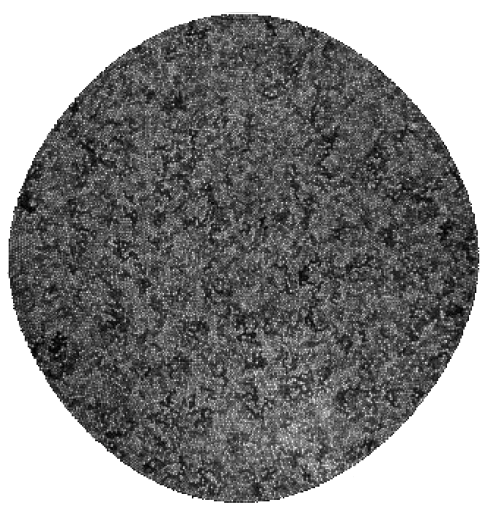

(b) [680-800]nm detection range mostly influenced by $660 \mathrm{~nm}$ excitation

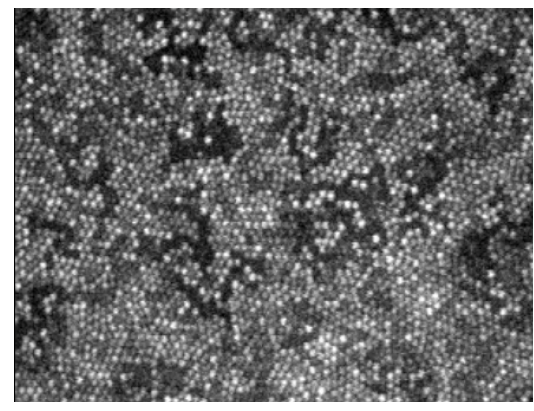

(e) Zoom on a portion of Fig.11b

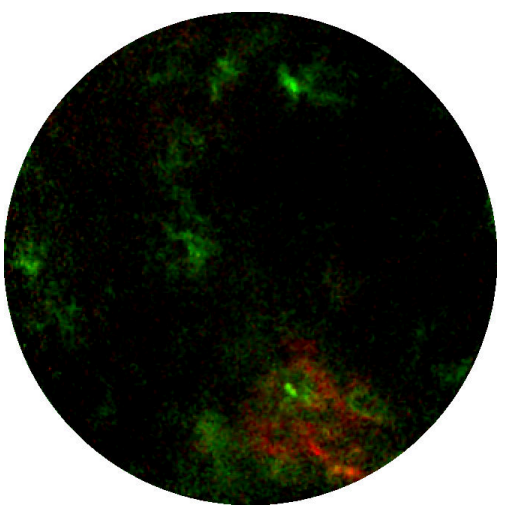

(c) Reconstruction and fusion of the signal estimated from the raw images in Fig.11a and Fig. $11 \mathrm{~b}$

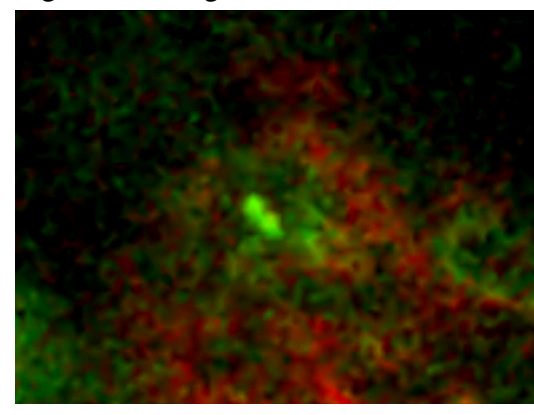

(f) Zoom on a portion of Fig. 11c

Figure 11: Typical example of the impact of the image reconstruction in the case of low tissue signal. We can clearly see the compensation of the geometric distortion. The fiber bundle does not appear as circular in Fig. 11a and Fig. 11b but does so after processing in Fig. 11c. It also appears that most of the signal observed after processing is hidden in the raw images by the honeycomb pattern.

Given, the relationship expressed in (3), our goal is to recover the tissue signal $u$ from the raw data on a per-fiber basis. To do this, we start from the detected fiber centers and build a mapping between the raw pixel locations and the fiber centers using a Voronoi diagram. This pixel-to-fiber mapping is then used to aggregate the signal of all pixels linked to a given fiber. This provides us with one scalar value per fiber and per spectral band that we refer to as the collected signal $v$. Specific calibration images are acquired in the air or water and in uniform medium. These calibration images are used to estimate the gains $\alpha$ and offsets $\beta$ of the photometric fiber transfer functions. Given these estimations, we can then invert equation (3) and obtain an estimate of the tissue signal $u$. From this information and the distortion-compensated fiber centers, we perform an interpolation to get a reconstructed image as illustrated in Fig. 11 
In more detail, the offsets $\beta$ of the fiber transfer functions can be decomposed as

$$
\beta_{i}^{k}=\sum_{l} I_{l} \cdot \tau_{i, l}^{\mathrm{inj}} \cdot \gamma_{i, l}^{k}
$$

where

- $l$ is an index for the lasers

- $I_{l}$ is the intensity of $l^{\text {th }}$ laser

- $\tau_{i n j}^{i, l}$ is the coupling efficiency of the $l^{\text {th }}$ laser within fiber $i$

- $\gamma_{i, l}^{k}$ is the propensity of fiber $i$ to emit a background signal when being excited by the $l^{\text {th }}$ laser. Concretely, $\gamma_{i, l}^{k}$ covers autofluorescence and Raman diffusion.

During the calibration procedure, we acquire several images by turning on one and only one laser at a time. As we have access, at all times, to the laser powers being used, this calibration data provide us with an estimate of $\left(\tau_{i, l}^{\mathrm{inj}} \cdot \gamma_{i, l}^{k}\right)$. It is therefore possible to update the offsets $\beta$ whenever the user changes the laser power during use.

The case of the gains $\alpha$ is different since we may not have access to calibration data that would allow for a proper decomposition. Indeed, the fluorophores may present different spectra in vivo and in a liquid solution or we may simply not have access to a uniform liquid solution composed of the fluorophores that will be observed in vivo. This will typically be the case when the fluorophores are endogenous or when the binding to other molecular species impacts either the absorption or the emission spectra. We therefore make the assumption that the fiber gains can be thought of as constant with respect to the laser powers. To avoid any confusion on the scale of the estimated tissue signal, we finally normalize the gain coefficients so as to have a unit median.

\section{IN VIVO APPLICATIONS}

The purpose of the experiments presented in this paper is to highlight the efficiency of our multispectral system to discriminate at least two labeling agents within a living animal. They therefore mostly serve as an in vivo technical validation and we refer the reader to other studies ${ }^{26134}$ for biologically, pre-clinically and clinically relevant evaluations.

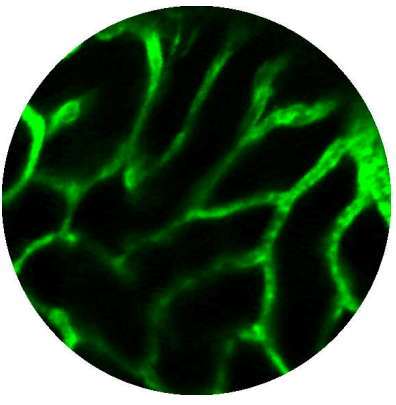

(a) [500-630]nm detection range mostly influenced by $488 \mathrm{~nm}$ excitation of FITCDextran

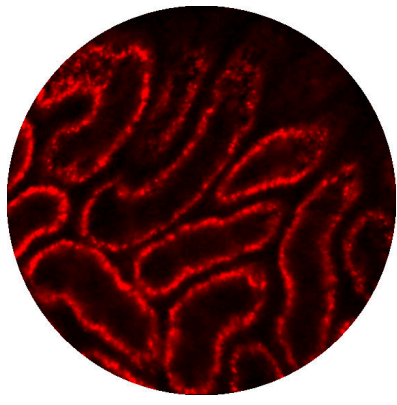

(b) $[680-800] \mathrm{nm}$ detection range mostly influenced by $660 \mathrm{~nm}$ excitation of Angiostamp

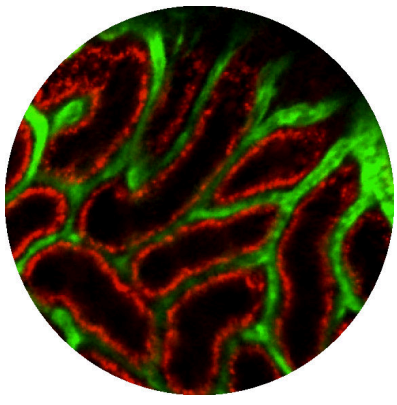

(c) Simple fusion of the images from the two spectral bands

Figure 12: Nude mouse kidney injected with FITC-Dextran and Angiostamp 700. The fused image clearly shows the perfect co-localization of the images taken at different spectral bands. The field of view is $240 \mu \mathrm{m}$

The imaging procedure with Cellvizio Dual Band was performed after the injection of at least two fluorescent dyes select among the following subsets.

With $488 \mathrm{~nm}$ excitation, Acriflavine labels the superficial epithelial cells, to stain the celllular architecture; FITC-Dextran (Fluorescein IsoThioCyanate labelled Dextran) can be used to perform blood stream visualization.

With $660 \mathrm{~nm}$ excitation, AminoSpark 680 (Perkin Elmer) accumulates in macrophages; AngioSense 680 (Perkin Elmer) remains localized in the vasculature; Angiostamp 700 (FluOptics) is designed for the in vivo assessment of angiogenesis. 


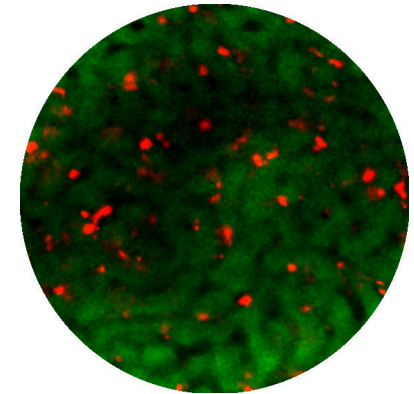

(a) Acryflavine and AminoSpark 680

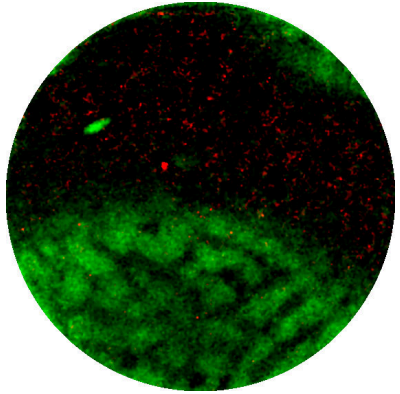

(b) Acryflavine and AngioSense 680

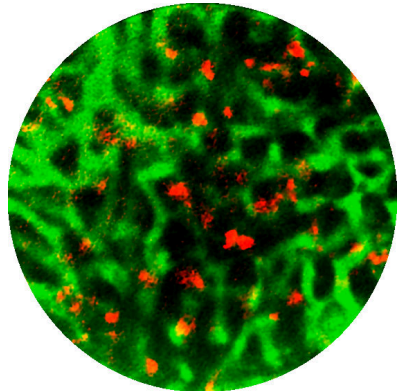

(c) FITC-Dextran and AminoSpark 680

Figure 13: Mouse liver imaged with different imaging markers. The field of view is $240 \mu \mathrm{m}$. All images are the result of the fusion of both spectral band

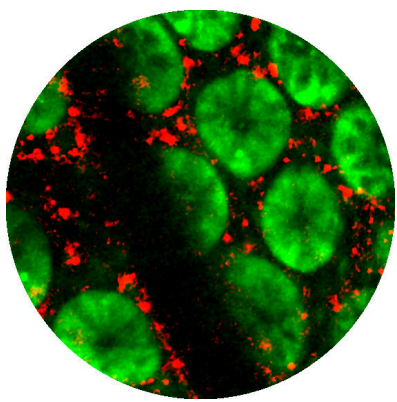

(a) Acryflavine

AminoSpark 680

and

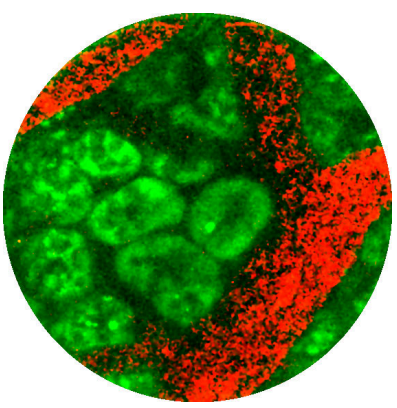

(b) Acryflavine and AngioSense 680

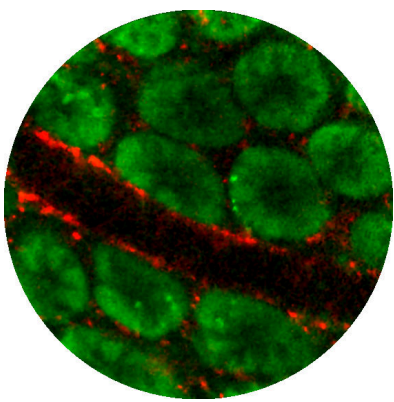

(c) Acryflavine, AminoSpark 680 and AngioSense 680

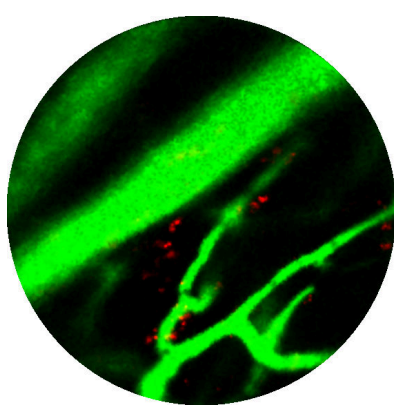

(d) $\quad$ FITC-Dextran AminoSpark 680

Figure 14: Mouse intestine imaged with different imaging markers. The field of view is $240 \mu \mathrm{m}$. All images are the result of the fusion of both spectral band

For the purpose of the first test, illustrated in Fig. 12, a nude mouse was used. This model is renown as the gold standard in cancer studies. Such rodent strains are immuno-compromised, meaning that they are lacking a functional immune system. Therefore, a tumor can develop easily within the animal, and since they are also lacking fur (nude), imaging procedures and experiments become much easier than for a standard animal.

To highlight the fact that our system is highly relevant for cancer-related studies, a fluorescent RGD-based marker, Angiostamp, was used. Angiostamp, binds the $\alpha_{v} \beta_{3}$ integrin and this protein is known to be overexpressed at the surface of some cancer cells as well as at the edge of endothelial cells during angiogenesis. Through a multimodal approach including X-ray computed tomography (CT) and fluorescence-enhanced diffuse optical tomography (fDOT), it was observed that Angiostamp can target a MDA-MB-231 tumor xenograft in vivo, that it is eliminated from the blood stream a few minutes after its injection, and that it accumulates close to the tumor blood vessels. 34

The mouse was anesthetized using isoflurane $1.5 \%$ administered through a nose cone. The blood flow and microvasculature were stained using high molecular weight Fluorescein-IsoThioCyanate labelled Dextran (FITC-Dextran, 500kDa), diluted in Phosphate Buffer Saline (PBS, 30mg/ml, 0.30ml), injected through the lateral tail vein of the mouse, 30 minutes before the imaging procedure. The choice of high weighted FITC-Dextran ensures a good control of the fluorescent signal leakage due to vessel permeability. Angiostamp 700 (FluOptics, $0.20 \mathrm{ml}, 500 \mu \mathrm{M}$ ) was inoculated via the lateral tail vein to the animal $7 \mathrm{~h} 30$ before the imaging procedure with the Cellvizio Dual Band.

Figure 12 underlines the excretion and elimination of both labeling agents used over the experiment. The inner structural anatomy of the kidney can be seen clearly and the dual color imaging reveals both anatomical and functional imaging of the organ at the same time.

For the purpose of the second test, illustrated in Fig. 13 to 15, standard wild mice were used with different imaging markers to illustrated to brode scope of possible applications of our system. In Fig. 13, we can see for example that both cells and vasculature of the liver can be easily observed with different combinations of fluorophores. Figure 14 shows that cells and 


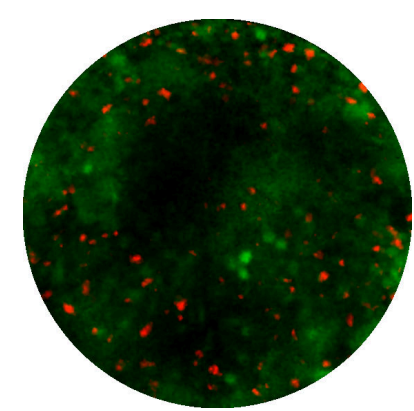

(a) Acryflavine and AminoSpark 680

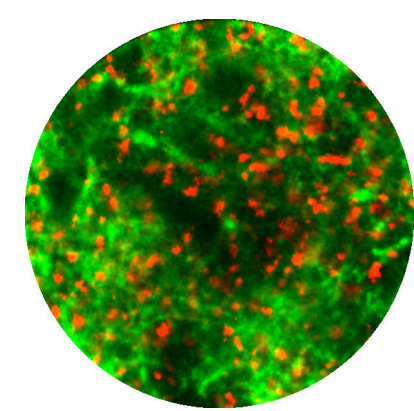

(b) FITC-Dextran and AminoSpark 680

Figure 15: Mouse spleen imaged with different imaging markers. The field of view is $240 \mu \mathrm{m}$. All images are the result of the fusion of both spectral band

vasculature in the GI tract can be examined in real-time. Figure 15 proves that organs and cells involved in inflammation processes can be examined by showing macrophages in the spleen with AminoSpark 680.

\section{DISCUSSION}

We showed in this paper how fibered-based confocal microscopy could be expanded to multicolor imaging. We have seen that this expansion was made possible not only by multiplying excitation sources and detection channels, but by redesigning the hardware and the software of the system, thus leading to a completely renewed architecture.

The particular context of in vivo applications, FRET, for instance, led us to prefer strictly simultaneous excitation and detection as a guarantee for unambiguous color to color 2D coregistration. In such a situation, spectral overlap between channels cannot be avoided and must be treated carefully to derive correct photometric quantitative data. From this standpoint, multispectral imaging is far from being just an expansion of one-color microscopy.

Our proposed architecture, on which is currently based the Cellvizio Dual Band product, has been designed to be modular, and so as to be easily expanded to simultaneous three colors fluorescence imaging, or a combination of fluorescence with reflectance microscopy. In the same way, spectroscopic capability can be added, which may be used in the future to complement visual interpretation and potentially improve sensitivity and specificity of clinical diagnosis.

This paper thus contributes to developing innovative research and diagnostic tools. In the current context of rapid development of molecular probes, allowing for highly specific targeting of biological entities, multispectral in vivo microscopy will certainly play a key role and pave the way to multimodal, morphological and functional, imaging.

\section{ACKNOWLEDGMENTS}

The authors would like to thank Bertrand Viellerobe for his visionary contributions and his system architecture skills and Frédéric Ducongé for performing the in vivo experiments from which our results in Fig. 12] are extracted.

\section{REFERENCES}

[1] Kiesslich, R., Goetz, M., Vieth, M., Galle, P. R., and Neurath, M. F., “Confocal laser endomicroscopy,” Gastrointest. Endosc. Clin. N. Am. 15, 715-731 (Oct. 2005).

[2] Wallace, M. and Fockens, P., "Probe-based confocal laser endomicroscopy," Gastroenterology 136, 1509-1513 (May 2009).

[3] Yun, S., Tearney, G., de Boer, J. F., Iftimia, N., and Bouma, B., "High-speed optical frequency-domain imaging," Opt. Express 11, 2953-2963 (Nov. 2003).

[4] Tearney, G. J., Webb, R. H., and Bouma, B. E., “Spectrally encoded confocal microscopy,” Opt. Lett. 23, 1152-1154 (Aug. 1998).

[5] Wang, T. D., Mandella, M. J., Contag, C. H., and Kino, G. S., "Dual-axis confocal microscope for high-resolution in vivo imaging," Opt. Lett. 28, 414-416 (Mar. 2003). 
[6] Sabharwal, Y. S., Rouse, A. R., Donaldson, L., Hopkins, M. F., and Gmitro, A. F., "Slit-scanning confocal microendoscope for high-resolution in vivo imaging," Appl. Opt. 38, 7133-7144 (Dec. 1999).

[7] Sung, K.-B., Liang, C., Descour, M., Collier, T., Follen, M., and Richards-Kortum, R., "Fiber-optic confocal reflectance microscope with miniature objective for in vivo imaging of human tissues," IEEE Trans. Biomed. Eng. 49, 1168-1172 (Oct. 2002).

[8] Laemmel, E., Genet, M., Le Goualher, G., Perchant, A., Le Gargasson, J.-F., and Vicaut, E., "Fibered confocal fluorescence microscopy (Cell-viZio ${ }^{\mathrm{TM}}$ ) facilitates extended imaging in the field of microcirculation," J. Vasc. Res. 41(5), 400-411 (2004).

[9] Tanbakuchi, A. A., Rouse, A. R., Udovich, J. A., Hatch, K. D., and Gmitro, A. F., "Clinical confocal microlaparoscope for real-time in vivo optical biopsies," J. Biomed. Opt. 14, 044030-044030-12 (Aug. 2009).

[10] Pierce, M. C., Schwarz, R. A., Bhattar, V. S., Mondrik, S., Williams, M. D., Lee, J. J., Richards-Kortum, R., and Gillenwater, A. M., "Accuracy of in vivo multimodal optical imaging for detection of oral neoplasia," Cancer Prev. Res. 5, 801-809 (June 2012).

[11] Becker, V., Vercauteren, T., von Weyern, C. H., Prinz, C., Schmid, R. M., and Meining, A., "High resolution miniprobe-based confocal microscopy in combination with video-mosaicing," Gastrointest. Endosc. 66, 1001-1007 (Nov. 2007).

[12] Thiberville, L., Moreno-Swirc, S., Vercauteren, T., Peltier, E., Cavé, C., and Bourg Heckly, G., "In vivo imaging of the bronchial wall microstructure using fibered confocal fluorescence microscopy,” Am. J. Respir. Crit. Care Med. 175, 22-31 (Jan. 2007).

[13] Flusberg, B. A., Cocker, E. D., Piyawattanametha, W., Jung, J. C., Cheung, E. L. M., and J., S. M., "Fiber-optic fluorescence imaging," Nat. Methods 2, 941-950 (Dec. 2005).

[14] Le Goualher, G., Perchant, A., Genet, M., Cavé, C., Viellerobe, B., Berier, F., Abrat, B., and Ayache, N., "Towards optical biopsies with an integrated fibered confocal fluorescence microscope,' Proc. MICCAI'04, Barillot, C., Haynor, D. R., and Hellier, P., eds., LNCS 3217, 761-768, Springer-Verlag (2004).

[15] Perchant, A., Le Goualher, G., Genet, M., Viellerobe, B., and Berier, F., "An integrated fibered confocal microscopy system for in vivo and in situ fluorescence imaging: Applications to endoscopy in small animal imaging," Proc. ISBI'04, 1, 692-695 (2004).

[16] Osdoit, A., Genet, M., Perchant, A., Loiseau, S., Abrat, B., and Lacombe, F., "In vivo fibered confocal reflectance imaging: totally non-invasive morphological cellular imaging brought to the endoscopist," Endoscopic Microscopy, Tearney, G. J. and Wang, T. D., eds., 6082(1), 608208-608210, SPIE, San Jose, CA, USA (2006).

[17] Viellerobe, B., Osdoit, A., Cavé, C., Lacombe, F., Loiseau, S., and Abrat, B., “Mauna Kea Technologies' F400 prototype: a new tool for in vivo microscopic imaging during endoscopy," Endoscopic Microscopy, Tearney, G. and Wang, T., eds., 6082(1), 60820c, SPIE (2006).

[18] Vercauteren, T., Ayache, N., Savoire, N., Malandain, G., and Perchant, A., "Processing of in vivo fibered confocal microscopy video sequences," [Microscopic Image Analysis for Life Science Applications], Rittscher, J., Machiraju, R., and Wong, S. T. C., eds., Artech House (2008). ISBN: 978-1-59693-236-4.

[19] André, B., Vercauteren, T., Buchner, A. M., Wallace, M. B., and Ayache, N., "A smart atlas for endomicroscopy using automated video retrieval," Med. Image Anal. 15(4), 460-476 (2011).

[20] Lacombe, F., Lavaste, O., and Senhadji, L., "Diagnostic precoce du cancer du côlon (early diagnosis of human colorectal cancer)," IRBM 32(2), 83-86 (2011).

[21] Kennedy, G., Manning, H., Elson, D., Neil, M., Stamp, G., Viellerobe, B., Lacombe, F., Dunsby, C., and French, P., "A fluorescence lifetime imaging scanning confocal endomicroscope," J. of Biophotonics 3(1-2), 103-107 (2010).

[22] Peyrot, D., Lefort, C., Mansuryan, T., Kruglik, S., Sandeau, N., Vever-Bizet, C., Thiberville, L., Lacombe, F., Louradour, F., and Bourg-Heckly, G., "Développement d'un endomicroscope non linéaire pour l'observation in vivo in situ de la matrice extracellulaire des tissus pulmonaires (development of a non-linear endomicroscope for the in vivo in situ observation of lung tissue extracellular matrix)," IRBM 33(2), 67-72 (2012).

[23] Kim, Y.-D., Ahn, M.-K., and Gweon, D.-G., "Design and fabrication of a multi-modal confocal endo-microscope for biomedical imaging,” J. Opt. Soc. Korea 15(3), 300-304 (2011).

[24] Makhlouf, H., Gmitro, A. F., Tanbakuchi, A. A., Udovich, J. A., and Rouse, A. R., "Multispectral confocal microendoscope for in vivo and in situ imaging," J. Biomed. Opt. 13(4), 044016 (2008). 
[25] Joshi, B. P., Miller, S. J., Lee, C. M., Seibel, E. J., and Wang, T. D., "Multispectral endoscopic imaging of colorectal dysplasia in vivo," Gastroenterology 143(6), 1435-1437 (2012).

[26] Barbosa-Brito, M., Guiot, E., Cavellini, L., Zhang, J., Ni, Q., Maskos, U., Paupardin-Tritsch, D., and Vincent, P., "Towards a real-time in vivo monitoring of PKA activity in deep brain regions using fibered fluorescence microscopy with dual emission detection," Proc. SFN'10, (Nov. 2010).

[27] Jean, F., Bourg-Heckly, G., and Viellerobe, B., "Fibered confocal spectroscopy and multicolor imaging system for in vivo fluorescence analysis," Opt. Express 15, 4008-4017 (Apr. 2007).

[28] Osdoit, A., Lacombe, F., Cavé, C., Loiseau, S., and Peltier, E., "To see the unseeable: confocal miniprobes for routine microscopic imaging during endoscopy," Endoscopic Microscopy, Tearney, G. and Wang, T., eds., 6432(1), 64320-64329, SPIE, San Jose, CA, USA (2007).

[29] Savoire, N., André, B., and Vercauteren, T., "Online blind calibration of non-uniform photodetectors: Application to endomicroscopy,” Proc. MICCAI'12, LNCS, 639-646, Springer-Verlag, Nice, France (Oct. 2012).

[30] Elter, M., Rupp, S., and Winter, C., "Physically motivated reconstruction of fiberscopic images," Proc. ICPR'06, 599-602 (Aug. 2006).

[31] Winter, C., Rupp, S., Elter, M., Münzenmayer, C., Gerhäuser, H., and Wittenberg, T., "Automatic adaptive enhancement for images obtained with fiberscopic endoscopes," IEEE Trans. Biomed. Eng. 53, 2035-2046 (Oct. 2006).

[32] Ford, T. N., Lim, D., and Mertz, J., "Fast optically sectioned fluorescence HiLo endomicroscopy,” J. Biomed. Opt. 17, 021105 (Feb. 2012).

[33] Besl, P. and McKay, H., "A method for registration of 3-D shapes," IEEE Trans. Pattern Anal. Mach. Intell. 14, 239-256 (Feb. 1992).

[34] Viellerobe, B., Janssens, I., Gombert, K., Gharbi, H., Lacombe, F., and Duconge, F., "Simultaneous imaging of two fluorescent signals using a new fibered fluorescent confocal microscopy system," Proc. WMIC'11, (Sept. 2011). 